\title{
Individual Variations in the Hardness and Elastic Modulus of the Human Cementum
}

\author{
Masaru Yamaguchi $^{1)}$, Eriko Yao-Umezawa ${ }^{1)}$, Yasuhiro Tanimoto ${ }^{2)}$, Mami Shimizu ${ }^{1)}$, Jun Kikuta ${ }^{1)}$, Takuji Hikida ${ }^{1}$, \\ Momoko Takahashi-Hikida ${ }^{1)}$, Satoshi Horihata ${ }^{3)}$, Kunihiro Suzuki ${ }^{4}$ and Kazutaka Kasai ${ }^{1)}$ \\ 1) Department of Orthodontics, Nihon University School of Dentistry at Matsudo, Chiba, Japan \\ 2) Department of Dental Biomaterials, Nihon University School of Dentistry at Matsudo, Chiba, Japan \\ 3) Department of Mathematical Science, Nihon University School of Dentistry at Matsudo, Chiba, Japan \\ 4) Department of Biology, Nihon University School of Dentistry at Matsudo, Chiba, Japan \\ (Accepted for publication, July 20, 2016)
}

\begin{abstract}
The purpose of this study was to measure the physical properties of enamel and cementum of permanent teeth and to determine the correlation between them. This study was conducted on 50 maxillary premolars that were collected from 26 orthodontic patients (10 males and 21 females, mean age: 19.51 years, range: 12-35 years). The hardness and elastic modulus of the enamel and cementum were measured at the surface of the crown and root at three locations (cervical third: CC, middle third: CM, and apical third: CA). There were individual differences in the hardness and elastic modulus of enamel and cementum. The hardness of the cementum decreased from the cervical to apical regions on the root surfaces in the moderate and soft groups $(\mathrm{p}<0.01)$. Individual variations were observed in the hardness and elastic modulus of the human first premolar cementum. A correlation was noted between the hardness and elastic modulus of the enamel and cementum in the CA group (hardness: $r=0.551, p<0.01$, elastic modulus: $\mathrm{r}=0.552, \mathrm{P}<0.01$ ). These results suggested that the physical properties of cementum may be involved in the occurrence of root resorption caused by orthodontic forces.
\end{abstract}

Key words: Hardness, Elastic modulus, Cementum, Enamel, Human teeth

\section{Introduction}

Orthodontically-induced inflammatory root resorption (OIIRR) is an unwanted side effect of orthodontic treatment. Motokawa et al. ${ }^{1}$ reported that teeth undergoing orthodontic treatment may develop some degree of OIIRR. It has been reported that $2 \%-5 \%$ of orthodontic patients experience more than $5 \mathrm{~mm}$ of resorption during orthodontic treatment with fixed appliances. It is therefore critical for orthodontists to anticipate this complication and prevent it whenever possible. The etiology of root resorption associated with orthodontic therapy is complex, and heavy force ${ }^{2)}$, lengthy treatment, and abnormal roots all further contribute to resorption ${ }^{3)}$.

Cementum contains $65 \%$ inorganic material and $12 \%$ water on a wet-weight basis. By volume, inorganic material comprises approximately $45 \%$, organic material $33 \%$, and water $22 \%$. Cementum is less densely mineralized than dentine and enamel and contains no blood vessels and does not undergo physiological remodeling ${ }^{4-6)}$. The chemical composition of cementum may vary

Correspondence to: Dr. Masaru Yamaguchi, Department of Orthodontics, Nihon University School of Dentistry at Matsudo, 2-870-1 SakaechoNishi, Matsudo, Chiba, 271-8587 Japan; Tel: +81-47-360-9414; Fax: +81-47-364-6295; E-mail: yamaguchi.masaru@nihon-u.ac.jp by individual, and morphologically, cementum is classified as both cellular and acellular ${ }^{7)}$.

In previous studies, Chutimanutskul et al. ${ }^{8)}$ examined the physical properties of the cementum on the buccal and lingual surfaces of the roots at the cervical third, middle third, and apical third. The authors reported a decreasing gradient in the hardness and elastic modulus of cementum in both surface groups, from the cervical to apical thirds. Apical cementum is predominately cellular, less densely mineralized and has lower hardness and elastic modulus values than the more densely mineralized acellular cementum found in the middle and cervical thirds of the root ${ }^{7}$. Therefore, the hardness and elastic modulus of cementum depend on the direction of the structural arrangement and the mineral content of the cementum ${ }^{9-11)}$. Several studies have reported that the hardness of mineralized tissues were positively correlated with the extent of mineralization ${ }^{12-14)}$. Some have suggested that the mineral content of cementum might influence the resistance or susceptibility to root resorption. Therefore, the hardness of cementum may be related to the incidence of OIIRR.

In the present study, we measured the physical properties of enamel and cementum of permanent teeth and examined the correlation between them. 


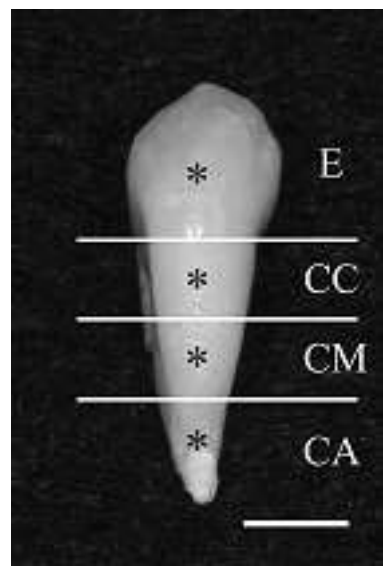

Figure 1. Location of indentations on maxillary premolar cementum and enamel.

Four positions on the buccal root surfaces were used. Scale bar: 5 $\mathrm{mm}$.

\section{Materials and Methods}

\section{Sample collection}

This study was conducted on 50 maxillary premolars collected from 26 orthodontic patients ( 10 males and 21 females, mean age: 19.51 years, range: $12-35$ years). We obtained informed consent from the patients, and the extracted teeth were used in accordance with a protocol reviewed by the Ethics Committee of Nihon University School of Dentistry at Matsudo (EC 15-001). The selection criteria were:

1. No previous reported or observed dental treatment of the teeth to be extracted,

2. No previous reported or observed trauma treatment of the teeth to be extracted,

3. No previous reported or observed orthodontic treatment of the teeth to be extracted,

4. No past or present signs or symptoms of periodontal disease,

5. No past or present signs or symptoms of bruxism,

6. No significant medical history that would affect the dentition,

7. No physical abnormalities concerning the anatomy of the craniofacial or dentoalveolar complex,

8. Completed apexification.

The teeth were extracted by oral surgeons who were asked to avoid damaging the cervical cementum with the forceps. Immediately after extraction, the teeth were stored in individual containers filled with sterilized deionized water. The extracted teeth were then placed in an ultrasonic bath for 10 minutes to remove the periodontal ligament (PDL) and soft tissue fragments. After the ultrasonic bath, the extracted teeth were gently swabbed with a damp gauze swab until all visible signs of the PDL had been removed. The extracted teeth were then sterilized in accordance with the protocol described in previous publications ${ }^{12)}$.

\section{Vickers hardness and elastic modulus tests}

We evaluated the Vickers hardness and elastic modulus of the extracted teeth's enamel and cementum and examined the correlation between these values for the enamel and apical third. The extracted teeth's roots were divided into equal thirds (cervical
Table 1. The mean and SD values of the hardness and elastic modulus of enamel and cementum

a) Vickers hardness

\begin{tabular}{lrrrr}
\hline & \multicolumn{1}{c}{ E } & \multicolumn{1}{c}{ CC } & \multicolumn{1}{c}{ CM } & \multicolumn{1}{c}{ CA } \\
\hline Mean & 394.46 & 26.50 & 20.67 & 19.00 \\
SD & 41.92 & 7.81 & 6.35 & 5.62 \\
Min. & 275.99 & 12.10 & 11.14 & 10.69 \\
Max. & 480.19 & 43.85 & 28.88 & 30.91 \\
\hline
\end{tabular}

b) Elastic modulus (GPa)

\begin{tabular}{lrrrr}
\hline & \multicolumn{1}{c}{ E } & \multicolumn{1}{c}{ CC } & \multicolumn{1}{c}{ CM } & \multicolumn{1}{c}{ CA } \\
\hline Mean & 82.00 & 11.67 & 9.13 & 7.26 \\
SD & 16.92 & 2.00 & 2.09 & 1.97 \\
Min. & 28.60 & 5.90 & 4.30 & 4.21 \\
Max. & 106.25 & 16.90 & 14.60 & 11.00 \\
\hline
\end{tabular}

$[\mathrm{CC}]$, middle $[\mathrm{CM}]$, and apical [CA]) for the measurements (Fig. 1). The measurement point was the center of the major axis in each region, at the center line on the buccal side. The mean values of three measurements in each part were obtained.

We focused on the CA Vickers hardness in particular, as root resorption following orthodontic treatment often occurs in the apical part. The extracted teeth were classified into soft, moderate, and hard groups according to the CA Vickers hardness as follows: soft group, less than the mean - 1 standard deviation (SD); moderate group, the mean $\pm \mathrm{SD}$; hard group, more than the mean $+1 \mathrm{SD}$. We compared the hardness of the $\mathrm{CC}, \mathrm{CM}$, and CA in each group.

The physical properties of the enamel and cementum were evaluated using a dynamic ultra-micro-hardness tester (DUH-211; Shimadzu Co., Kyoto, Japan) with a load rate of $13.32 \mathrm{mN} / \mathrm{s}$ and a holding time of $10 \mathrm{~s}$ at a maximum load of $98.07 \mathrm{mN}$.

\section{Statistical analysis}

In the statistical analysis, we compared the mean CC, CM, and CA hardness values of each group using the Steel-Dwass test, with significance set at $\mathrm{P}<0.01$. A box plot analysis was used to determine the hardness and elastic modulus of the enamel and three regions ( $\mathrm{CC}, \mathrm{CM}, \mathrm{CA})$ in the cementum. Correlation coefficients and regression equations were formulated to evaluate any relationship between the physical properties of the enamel and $\mathrm{CA}$.

\section{Results}

The mean and SD values of the hardness and elastic modulus of the enamel and cementum are shown in Table 1. A box plot analysis revealed significant individual variations in the mean hardness and elastic modulus of the cementum (respective ranges: 10.69-43.85 and 4.21-16.90 GPa) and enamel (respective ranges: 275.99-480.19 and 28.60-106.25 GPa) (Figs. 2-a, b, 3-a, b). The 
Masaru Yamaguchi et al.: Hardness and Elastic Modulus in Human Cementum

a

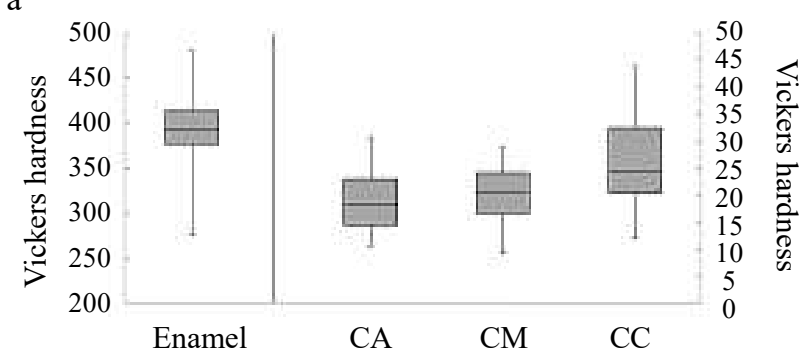

b

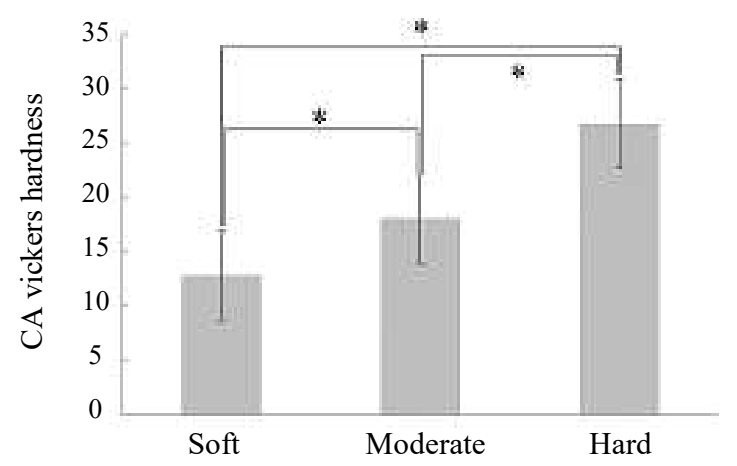

Figure 2. The hardness of enamel and cementum.

a: Box plot analysis of the hardness of enamel and cementum (CC, $\mathrm{CM}$ and $\mathrm{CA})$.

b: Significant differences were noted among the soft, moderate, and hard group. $(* \mathrm{P}<0.01$ from the Steel-Dwass test). a

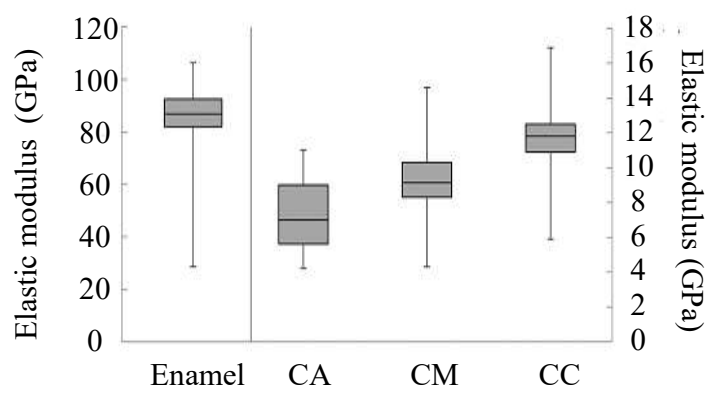

b

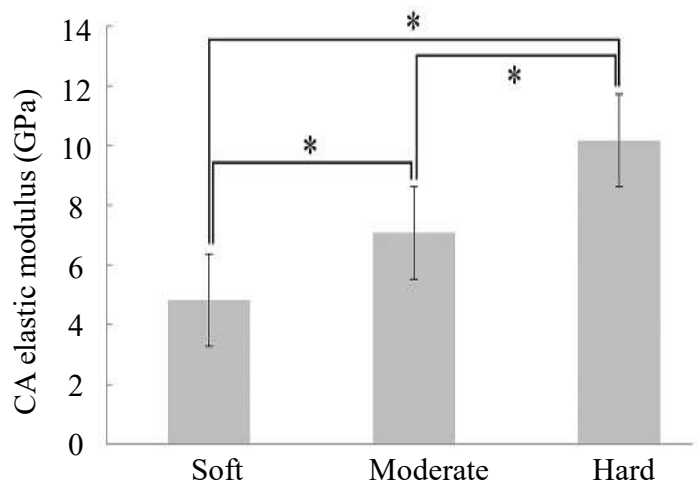

Figure 3.The elastic modulus of enamel and cementum.

a: Box plot analysis of the elastic modulus of enamel and cementum (CC, CM and CA).

b: Significant differences were noted among the soft, moderate, and hard group. $(* \mathrm{P}<0.01$ from the Steel-Dwass test).

Table 2. Comparison of the hardness and elastic modulus of the CC, CM, and CA in each group

a) Vickers hardness

\begin{tabular}{|c|c|c|c|c|c|c|c|c|c|}
\hline & \multicolumn{2}{|c|}{$\mathrm{CC}$} & \multicolumn{2}{|c|}{$\mathrm{CM}$} & \multicolumn{2}{|c|}{$\mathrm{CA}$} & \multicolumn{3}{|c|}{ Steel-Dwass } \\
\hline & Mean & SD & Mean & SD & Mean & SD & $\mathrm{CC}$ vs $\mathrm{CM}$ & $\mathrm{CC}$ vs $\mathrm{CA}$ & $\mathrm{CM}$ vs $\mathrm{CA}$ \\
\hline Total & 26.50 & 7.81 & 20.67 & 6.35 & 19.00 & 5.62 & $*$ & $*$ & NS \\
\hline Soft & 24.11 & 5.62 & 21.25 & 9.28 & 12.84 & 1.26 & NS & $*$ & $*$ \\
\hline Moderate & 25.76 & 6.36 & 19.72 & 5.06 & 18.09 & 2.54 & $*$ & $*$ & NS \\
\hline Hard & 30.28 & 10.84 & 21.86 & 5.06 & 26.85 & 2.37 & NS & NS & NS \\
\hline
\end{tabular}

$* \mathrm{P}<0.01$, NS: not significant.

b) Elastic modulus (GPa)

\begin{tabular}{|c|c|c|c|c|c|c|c|c|c|}
\hline & \multicolumn{2}{|c|}{$\mathrm{CC}$} & \multicolumn{2}{|c|}{$\mathrm{CM}$} & \multicolumn{2}{|c|}{$\mathrm{CA}$} & \multicolumn{3}{|c|}{ Steel-Dwass } \\
\hline & Mean & SD & Mean & SD & Mean & SD & $\mathrm{CC}$ vs $\mathrm{CM}$ & $\mathrm{CC}$ vs $\mathrm{CA}$ & $\mathrm{CM}$ vs $\mathrm{CA}$ \\
\hline Total & 11.67 & 2.00 & 9.13 & 2.09 & 7.26 & 1.97 & $*$ & $*$ & $*$ \\
\hline Soft & 11.59 & 1.84 & 9.27 & 2.11 & 5.53 & 1.09 & * & $*$ & $*$ \\
\hline Moderate & 11.12 & 1.82 & 8.64 & 2.08 & 7.15 & 1.53 & $*$ & $*$ & NS \\
\hline Hard & 12.87 & 2.13 & 9.94 & 1.97 & 9.41 & 1.58 & * & * & NS \\
\hline
\end{tabular}

$* \mathrm{P}<0.01$, NS: not significant.

hardness and elastic modulus of the cementum in the CM and CA were lower than in the CC (Table 1-a, b). No significant differences in the values were noted between genders (date not shown).

Regarding the CA hardness of the three groups (soft, moderate, and hard), the hardness decreased from the cervical to apical regions on the root surfaces in the soft and moderate groups $(p<0.01)$, while the differences in the hardness of different regions in the hard group were not significant (Table 2-a). The elastic modulus decreased from the cervical to apical regions on the root surfaces in all hardness groups $(\mathrm{p}<0.01)$ (Table 2-b).

To estimate the physical properties of the cementum based on those of the enamel, we examined the correlation between them and found a positive correlation between the hardness and elastic modulus of the enamel and cementum in the CA (hardness: $\mathrm{r}=0.551, \mathrm{p}<0.01$, elastic modulus: $\mathrm{r}=0.552, \mathrm{P}<0.01)$ (Figs. 4,5$)$. 


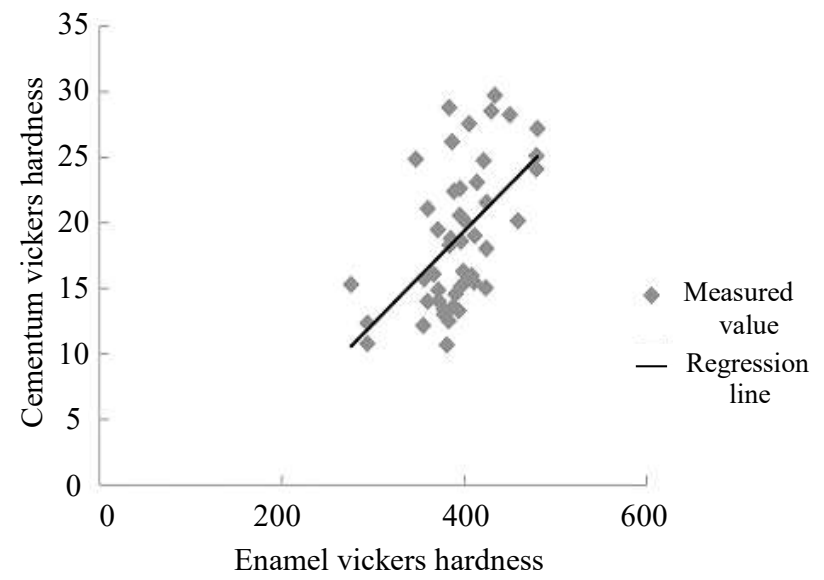

Figure 4. Correlation between the hardness of the enamel and cementum.

A correlation was shown between the hardness of the enamel and cementum in the CA group $(\mathrm{r}=0.551, \mathrm{P}<0.01)$.

\section{Discussion}

Hardness is a major parameter to consider when observing structural changes in the cementum tissue that depend on root resorption caused by orthodontic forces. In this study, the dynamic micro-indentation method was applied to investigate mechanical properties such as the indentation hardness and elastic modulus of the enamel and cementum.

Generally, there are two methods used to measure the indentation hardness of materials. The conventional procedure for testing hardness involves applying a fixed load to a diamond indenter and measuring, with the aid of optical microscopy, the dimensions of the resultant indentation on the surface of the test material after unloading. However, it is difficult to optically measure such dimensions with a high degree of accuracy for indents in micro-scale structures, such as biotissue. The dynamic micro-indentation method therefore has significant advantages over conventional hardness testing. This method is a depth-sensing technique that can accurately characterize the mechanical properties of almost all types of solid materials on a small scale. In other words, this method can continuously record the penetration depth of the cementum tissue during small dynamic loading and unloading at the indentation tip while providing information on the hardness and elastic modulus of the small contact surface.

The present study examined whether or not there was individual variation in the hardness and elastic modulus values of the cementum and enamel of permanent teeth. Regarding the age of the samples in this study, Pinchi et al. ${ }^{15)}$ reports that the cementum thickens with age. We therefore were limited in the age range of the samples we could use without thickening of the cementum (12-35 years old). Furthermore, there was no significant difference in the values of gender (date not shown).

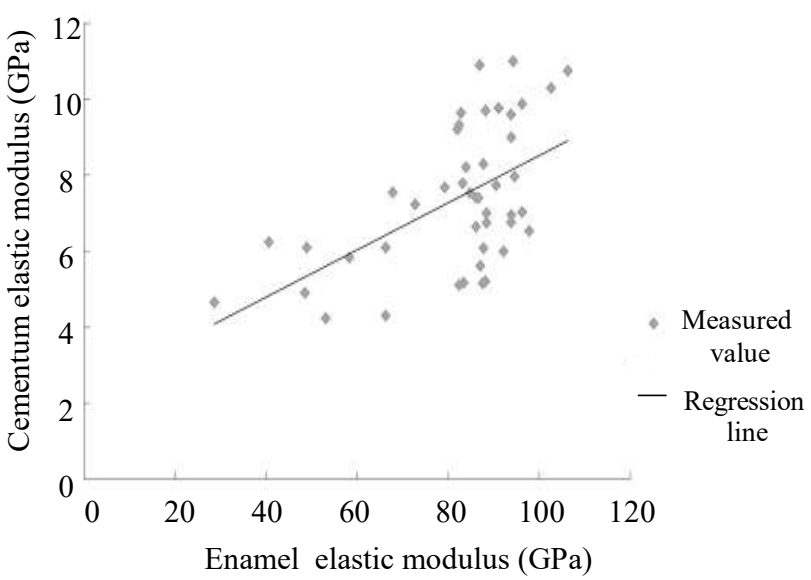

Figure 5. Correlation between the elastic modulus of enamel and cementum.

A correlation was shown between the elastic modulus of the enamel and cementum in the $\mathrm{CA}$ group $(\mathrm{r}=0.552, \mathrm{P}<0.01)$.

The results in this study showed that the respective hardness and elastic modulus of the enamel and cementum differed significantly. (Figs. 2-a, b, 3-a, b, Table 1-a,b). Relatively little is known about the individual variation in the physical properties of the enamel and cementum of permanent teeth. In their examination of a small ( $n=16)$ mixed sample of maxillary and mandibular first premolars, Darendeliler et al. ${ }^{16)}$ reported large individual variation for both the hardness and elastic modulus. These present and previous findings support the existence of individual differences in the hardness and elastic modulus of the cementum.

Generally, the mineralized surface of the cementum in humans is more resorbed in the apical part after orthodontic tooth movement than in other regions. Therefore, we measured the hardness and elastic modulus in the CA. On dividing the CA hardness into three groups (soft, moderate, and hard), we found that the hardness decreased from the cervical to apical regions on the root surfaces in the soft and moderate groups ( $p<0.01$ ), while the differences in the hardness of different regions in the hard group were not significant (Table 2-a). Furthermore, the elastic modulus decreased from the cervical to apical regions on the root surfaces in all hardness groups $(\mathrm{p}<0.01)$ (Table 2-b).

Chutimanutskul et al. ${ }^{8)}$ reported that the hardness and elastic modulus of the human maxillary first premolar cementum gradually decreased from the cervical to apical regions on the buccal and lingual surface. The cervical two thirds of the root is covered by acellular cementum that consists of mineralized layers. However, the apical cementum is predominately cellular cementum. The hardness and elastic modulus of the apical region are lower than those of the middle and cervical thirds of the root. Rex et al. ${ }^{11)}$ demonstrated that there were large individual variations in the hardness of the cementum of the first premolars based on the calcium $(\mathrm{Ca})$, phosphorus $(\mathrm{P})$, and fluorine $(\mathrm{F})$ concentrations, 
Masaru Yamaguchi et al.: Hardness and Elastic Modulus in Human Cementum

with a decreasing gradient in these concentrations from the cervical to the apical third of the root. These previous findings may support the results in the present study. Taken together, these present and previous findings suggest that the low hardness value (soft) may be due to low concentrations of $\mathrm{Ca}, \mathrm{P}$, and $\mathrm{F}$ and be involved in the outbreak of OIIRR in the apical region. Preparations are currently underway for a new study measuring the proportions of $\mathrm{Ca}, \mathrm{P}$, and $\mathrm{F}$ in the apical zone in the soft, moderate, and hard groups.

Finally, we observed a positive correlation between the respective hardness and elastic modulus of the enamel and cementum in the CA group (hardness: $\mathrm{r}=0.551, \mathrm{p}<0.01$, elastic modulus: $\mathrm{r}=0.552, \mathrm{P}<0.01$ ) (Figs. 4,5 ). Fong et al. ${ }^{17)}$ showed that enamel-related proteins such as amelin were associated with root formation in rats. Tokiyasu et al. ${ }^{18)}$ showed that the enamel matrix derivative activated cementoblasts in vivo and in vitro. Previous studies have identified amelogenin as an enamel protein associated with enamel formation application that suppresses root resorption by inhibiting odontoclast number in vitro ${ }^{19)}$. Therefore, these factors involved in enamel formation may not only influence the formation of cementum, but also odontoclast activities in root resorption.

Although root abnormalities is a risk factor of OIIRR ${ }^{20)}$, an apparently normal root can resorb severely during orthodontic treatment. This phenomenon may be associated with the hardness and elastic modulus of the cementum. If the cementum hardness and elastic modulus can be estimated based on the enamel value, we may be able to prevent OIIRR. Further studies will be necessary to clarify the relationship between the enamel and cementum with respect to the mechanical properties.

\section{Acknowledgments}

This study was supported by a Grant-in-Aid For Scientific Research from the Japan Society for the Promotion of Science (C:25463200, C:26893289, C:26440127, 15H06648, 16K11795 and $16 \mathrm{~K} 20656)$.

\section{Conflict of Interest}

The authors have declared that no competing interest exists.

\section{References}

1. Motokawa M, Sasamoto T, Kaku M, Kawata T, Matsuda Y, Terao A and Tanne K. Association between root resorption incident to orthodontic treatment and treatment factors. Eur J Orthod 34: 350-356, 2012

2. Chan E and Darendeliler MA. Physical properties of root cementum: Part 5. Volumetric analysis of root resorption craters after application of light and heavy orthodontic forces. Am J Orthod Dentofacial Orthop 127: 186-195, 2005

3. Levander E, Malmgren O and Stenback K. Apical root resorption during orthodontic treatment of patients with multiple aplasia: a study of maxillary incisors. Eur J Orthod 20: 57-63, 1998

4. Cohen M, Garnick JJ, Ringle RD, Hanes PJ and Thompson WO. Calcium and phosphorus content of roots exposed to the oral environment. J Clin Periodontol 19: 268-273, 1992

5. Neiders ME, Eick JD, Miller WA and Leitner JW. Electron probe microanalysis of cementum and underlying dentin in young permanent teeth. J Dent Res 51:122-130, 1972

6. Selving KA and Selving SK. Mineral content of human and seal cementum. J Dent Res 41: 624-632, 1962

7. Ten cate AR. Oral Histology, Development, Structure, and Function. 5 th ed. 253-288 St. Louis: Mosby, 1998

8. Chutimanutskul W, Darendeliler MA, Swain MV, Shen G and Petocz P. Physical properties of human premolar cementum: hardness and elasticity. Aust Orthod J 21:117121, 2005

9. Henry JL and Weinmann JP. The pattern of resorption and repair of human cementum. J Am Dent Assoc 42:270-290, 1951

10. Jones SJ and Boyde A. A study of human root cementum surfaces as prepared for and examined in the scanning electron microscope. Z Zellforsch Mikrosk Anat 130: 318 337,1972

11. Rex T, Kharbanda OP, Petocz P and Darendeliler MA. Physical properties of root cementum: Part 4. Quantitative analysis of the mineral composition of human premolar cementum. Am J Orthod Dentofacial Orthop 127:177-185, 2005

12. Malek S, Darendeliler MA and Swain MV. Physical properties of root cementum: Part I. A new method for 3-dimensional evaluation. Am J Orthod Dentofacial Orthop 120: 198-208, 2001

13. Mahoney E, Holt A, Swain M and Kilpatrick N. The hardness and modulus of elasticity of primary molar teeth: an ultramicro-indentation study. J Dent 28:589-594, 2000

14. Brear K, Currey JD, Pond CM and Ramsay MA. The mechanical properties of the dentine and cement of the tusk of the narwhal Monodon monoceros compared with those of other mineralized tissues. Arch Oral Biol 35: 615-621, 1990

15. Pinchi V, Forestieri AL and Calvitti M. Thickness of the dental (radicular) cementum: a parameter for estimating age. J Forensic Odontostomatol 25: 1-6, 2007

16. Darendeliler MA, Kharbanda OP, Chan EK, Srivicharnkul P, Rex T, Swain MV, Jones AS and Petocz P. Root resorption and its association with alterations in physical properties, mineral contents and resorption craters in human premolars following application of light and heavy controlled orthodontic forces. Orthod Craniofac Res 7: 79-97,2004 
17. Fong CD, Slaby I and Hammarström L. Amelin: an enamelrelated protein, transcribed in the cells of epithelial root sheath. J Bone Miner Res 11: 892-898, 1996

18. Tokiyasu Y, Takata T, Saygin E and Somerman M. Enamel factors regulate expression of genes associated with cementoblasts. J Periodontol 71(12): 1829-1839, 2000
19. Yagi Y, Suda N, Yamakoshi Y, Baba O and Moriyama K. In vivo application of amelogenin suppresses root resorption. J Dent Res 88: 176-181, 2009

20. Maués CP, Nascimento RR and Vilella OV. Severe root resorption resulting from orthodontic treatment: prevalence and risk factors. Dental Press J Orthod 20: 52-58, 2015 